\title{
Dietary patterns and sleep disorders in Mexican adults from a National Health and Nutrition Survey
}

\author{
Elsa B. Gaona-Pineda ${ }^{1}$ (D), Brenda Martinez-Tapia ${ }^{1}$ (D), Sonia Rodríguez-Ramírez ${ }^{2}$ (), Selene Guerrero- \\ Zúñiga $^{3}$ (D), Rogelio Perez-Padilla ${ }^{3}$ (i) and Teresa Shamah-Levy ${ }^{1}$ (1) \\ ${ }^{1}$ Center for Evaluation and Surveys Research, National Institute of Public Health, Av. Universidad 655, Santa Maria Abuacatitlán, Cuernavaca, \\ Morelos 62100, Mexico \\ ${ }^{2}$ Center for Nutrition and Health Research, National Institute of Public Health, Cuernavaca, Morelos, Mexico \\ ${ }^{3}$ Mexican National Institute of Respiratory Diseases, Mexico City, Mexico
}

(Received 28 November 2020 - Final revision received 3 April 2021 - Accepted 14 April 2021)

Journal of Nutritional Science (2021), vol. 10, e34, page 1 of 10

doi:10.1017/jns.2021.24

Abstract

Given the high prevalence of multiple non-communicable chronic diseases in Mexico, the aim of the present study was to assess the association between dietary patterns and sleep disorders in a national representative sample of 5076 Mexican adults (20-59 years) from the 2016 National Health and Nutrition Survey. Through a cross-sectional study, we used the Berlin sleep symptoms questionnaire to estimate the proportion of adults with insomnia, obstructive sleep apnoea (OSA) and other related problems such as daytime symptoms and inadequate sleep duration. Dietary data were collected through a seven-day semi-quantitative food frequency questionnaire, and dietary patterns were determined through cluster analysis. Associations between dietary patterns and sleep disorders were assessed by multivariate logistic regression models adjusted for age, sex, well-being, rural/urban area type, geographical region, tobacco use, physical activity level and energy intake. Three dietary patterns were identified: traditional (high in legumes and tortilla), industrialised (high in sugarsweetened beverages, fast foods, and alcohol, coffee or tea) and mixed (high in meat, poultry, fruits and vegetables). Multivariate logistic regression showed that the industrialised pattern yielded higher odds for daytime symptoms (OR 1.49; $95 \%$ CI 1.12, 1.99) and OSA (OR 1.63; 95 \% CI 1.21, 2.19) compared with the traditional pattern. In conclusion, dietary patterns are associated with sleep disorders in Mexican adults. Further research is required to break the vicious cycle of poor-quality diet, sleep symptoms and health.

Key words: Dietary patterns: Health surveys: Mexico: Obstructive sleep apnoea: Sleep quality

\section{Introduction}

Epidemiological evidence suggests that sleep disorders are associated with adverse health conditions ${ }^{(1)}$. According to the National Sleep Foundation, a duration of 7-9 h of sleep are necessary for optimal health in adults (26-64 years of age $)^{(2)}$. Less than $6 \mathrm{~h}$ is insufficient and has been associated with obesity $^{(3)}$, hypertension ${ }^{(4)}$, type 2 diabetes $^{(5)}$, cardiovascular disease (CVD) ${ }^{(6)}$ and all-cause mortality ${ }^{(7)}$.

Insomnia is one of the most common sleep disorders. It is characterised by difficulty initiating or maintaining sleep, premature awakening and non-restorative sleep ${ }^{(8)}$, accompanied by negative daytime consequences in social, occupational and behavioural performance as well as in other important life functions. These symptoms have been associated with mortality $^{(9,10)}, \mathrm{CVD}^{(11)}$ and injury ${ }^{(12)}$.

Obstructive sleep apnoea (OSA), another prevalent sleep disorder, is defined by repetitive episodes of apnoea (cessation of inspiratory airflow during sleep which lasts $\geq 10 \mathrm{~s}$ ) or hypopnoea (a reduction of $\geq 30 \%$ in inspiratory airflow lasting $\geq 10 \mathrm{~s}$, with an associated drop in oxygen saturation or arousal

* Corresponding author: Teresa Shamah-Levy, email tshamah@insp.mx

(C) The Author(s), 2021. Published by Cambridge University Press on behalf of The Nutrition Society. This is an Open Access article, distributed under the terms of the Creative Commons Attribution licence (http://creativecommons.org/licenses/by/4.0/), which permits unrestricted re-use, distribution, and reproduction in any medium, provided the original work is properly cited. 
from sleep $)^{(13)}$. This condition has been associated with many different forms of CVD including hypertension, stroke, heart failure, coronary artery disease and atrial fibrillation. Adults with OSA face not only an increased risk of developing comorbid CVD but also worse CVD-related outcomes ${ }^{(14)}$.

One of the primary symptoms associated with sleep disorders is excessive daytime sleepiness (EDS) ${ }^{(15)}$. This condition reduces performance in school ${ }^{(16)}$ or at work ${ }^{(17,18)}$ due to concentration, memory and mood problems ${ }^{(19)}$.

Given their role in adverse health outcomes, the causes of sleep disorders have been studied from various perspectives, including through diet. It has been reported that adequate sleep is positively associated with a healthy diet in children ${ }^{(20)}$, adolescents ${ }^{(21-23)}$ and adults ${ }^{(24-26)}$. Conversely, individuals with insufficient sleep duration are more likely to have lower-quality diets and adopt irregular meal patterns. They tend to consume more energy-rich foods, calories, fats, refined carbohydrates and snacks, and fewer proteins, fruits and vegetables ${ }^{(27,28)}$.

A study of Japanese workers found that a healthy dietary pattern (characterised by vegetables, mushrooms, potatoes, seaweed, soya products and eggs) was associated with a decreased risk of difficulty initiating sleep ${ }^{(29)}$. By contrast, a high intake of processed meat, red meat, snacks and take-out food was positively associated with the apnoea-hypopnoea index score after adjusting for demographic and lifestyle factors. Another study in Australia found that those who slept less time exhibited higher energy intake, especially from snacks and foods containing fat ${ }^{(30)}$.

Among middle-aged female Japanese workers, low protein intake was shown to be associated with difficulty initiating sleep, while high protein and low carbohydrate intake were associated with difficulty maintaining sleep ${ }^{(31)}$, and high carbohydrate intake with poor sleep quality ${ }^{(32)}$.

Few reports exist which document sleep disorders in Mexico, and those published only take place in Mexico City ${ }^{(33,34)}$ or with elderly participants ${ }^{(35)}$. The 2016 National Health and Nutrition Survey was the first study to reveal a high prevalence of multiple sleep disorders in the Mexican adult population: $28.4 \%$ reported short sleep duration $(<7 \mathrm{~h}), 18.8 \%$ reported insomnia and $27.3 \%$ showed high risk for $\mathrm{OSA}^{(36)}$. Furthermore, few studies have related diet with sleep disorders in Mexican adults. In one adolescent cohort, consumption of a healthy plant-based and lean protein dietary pattern was associated with an earlier bedtime, as assessed 2 years later ${ }^{(37)}$. In one cohort of female teachers, a fruit and vegetable-based dietary pattern was associated with greater sleep quality ${ }^{(38)}$. Given the documented relationship between sleep alterations and adverse health outcomes, the aim of the present study was to analyse the association between dietary patterns and sleep disorders in Mexican adults (age 2059) based on data from a nationally representative survey.

\section{Methods}

The 2016 National Health and Nutrition Survey (in Spanish, ENS ANUT-2016) is a cross-sectional survey with a probabilistic and multistage sampling design. The data collected allows statistical inferences regarding health and nutrition in the Mexican population at the national, geographical region and urban/rural area levels ${ }^{(39)}$. We analysed a sample of adults aged 20-59 through information regarding sleep and dietary habits.

\section{Sleep disorders}

The ENSANUT-2016 surveyed a sample of the Mexican adult population aged $\geq 20$ using the Berlin questionnaire on OSA risk, sleep duration, insomnia and $\operatorname{EDS}^{(36)}$. Average sleep duration over the 6 months before interview date was categorised as: $<7 \mathrm{~h}, 7-9 \mathrm{~h}$ and $>9 \mathrm{~h}^{(40)}$. Insomnia was defined as waking up prematurely or experiencing difficulty initiating or maintaining sleep $\geq 3-4$ times per week within the last 3 weeks ${ }^{(41)}$. Daytime symptoms were defined as the perception of not feeling rested or feeling sleepy/tired during the day $\geq 3 \mathrm{~d} /$ week.

Participants with affirmative scores in two or more of the following symptom categories were classified as high-risk for OSA: ${ }^{(42)}$

Category 1. Nighttime symptoms were explored through five questions, with responses assigned the following values: snoring $=1$ point; snoring loud or very loud $=1$ point; snoring $\geq 3 \mathrm{~d} /$ week $=1$ point; snoring in a way that has annoyed others $=1$ point; and another person noticing the cessation of the respondent's breathing during sleep $\geq 3 \mathrm{~d}$ /week $=2$ points. An affirmative result in this category was a score of $\geq 2$ points. Category 2. Daytime sleepiness was explored through three questions, with responses assigned the following values: the perception of non-restorative sleep $\geq 3 \mathrm{~d} /$ week $=1$ point, daytime tiredness $\geq 3 \mathrm{~d} /$ week $=1$ point and somnolence while driving $=1$ point. An affirmative result in this category was a score of 2 points.

Category 3. An affirmative result in this category was considered as suffering from obesity or hypertension.

\section{Dietary intake data and dietary patterns}

Dietary information was obtained from 6511 adults through a semi-quantitative food frequency questionnaire (SFFQ) on consumption during the $7 \mathrm{~d}$ before the survey. The SFFQ included a list of 140 foods and beverages. Consumption was estimated in grams based on the following parameters: number of days, times per day, portion size, number of portions consumed, edible portions (for meat, poultry, fruits and vegetables) and density (for beverages). Consumption data $>4$ sD from the mean by food or beverage item were considered implausible and those values were imputed with the average consumption by sex, urban/rural area and region (North, Centre, Mexico City and South). Participants with $>7$ food and beverage items with consumption imputations were excluded from analysis $(n 8)$. Energy intake was estimated according to the nutrient database compiled by the National Institute of Public Health of Mexico (unpublished material). Energy intake requirements were estimated based on equations from the Institute of Medicine, considering sex, age, height and weight ${ }^{(43)}$ and the Mifflin-St Jeor equation was used to estimate resting metabolic rate ${ }^{(44)}$. Results showing the ratios of energy intake/energy requirement $>3$ SD from the average, by sex, were excluded from analysis, as were those which indicated an energy intake of less than half of the resting metabolic rate $(n$ 161). 
Foods and beverages were classified into twenty-six food groups according to their nutritional content. In addition, a separate food group included alcoholic and caffeinated beverages such as coffee and tea, due to their demonstrated relationship with sleep disorders ${ }^{(45,46)}$ (Table 1). Dietary patterns were determined through $\mathrm{k}$-means cluster analysis using the standardised percentage of contribution to energy intake of each food group ${ }^{(47)}$. Two to five clusters were specified within the k-means analysis in order to evaluate which set of clusters discriminated better across groups, while still maintaining an adequate sample size within each cluster.

\section{Covariables}

The following variables were drawn from a health items questionnaire: lifetime tobacco use $(>100, \leq 100 \text { or } 0 \text { cigarettes })^{(48)}$ and previous diagnosis of hypertension. The variables of age, sex and well-being index (WBI) were drawn from a sociodemographic questionnaire. WBI was estimated through principal component analysis based on housing characteristics (roof, wall and flooring materials; drainage; water provision) and material possessions (television, computer, radio, telephone, cable television, refrigerator, microwave, stove, boiler, washing machine). The percentage of variance explained by the first component was $40 \%$. WBI was categorised into tertiles, with tertile 1 being low and 3 being high.

Overweight and obesity were determined by body mass index (BMI) (weight in kilograms/height in meters ${ }^{2}$ ). BMI was categorised according to the cut-off points established by the World Health Organization (WHO) ${ }^{(49)}$. Also in line with WHO recommendations, physical activity was estimated using the International Physical Activity Questionnaire (IPAQ) validated in the Mexican adult population ${ }^{(50)}$.

\section{Statistical analysis}

We estimated proportions and means of the categorical and continuous variables, respectively, with $95 \%$ confidence intervals (95\% CIs). Comparison among groups was performed through linear, logit or quantile regression, depending on the variable type. The Bonferroni method was applied to adjust $P$-value ${ }^{(51)}$. Logistic regression models were used to assess the association between dietary patterns and each sleep disorder, and models were adjusted by age, sex, WBI, rural/ urban area, geographical region, tobacco use, physical activity level, total energy intake and BMI (with the exception of the OSA logistic regression model, since obesity defined as BMI $\geq 30 \mathrm{~kg} / \mathrm{m}^{2}$ is included in the classification criteria for highrisk OSA). All logistic regression models were stratified by sex. Sensitivity analyses were performed accounting for use of sedative medications. All statistical analyses were performed using STATA 14.0 with survey commands (SVY).

\section{Results}

We analysed data from 5076 adults who answered the sleep questionnaire described earlier and who indicated biologically plausible dietary intakes. The sample represented 59251709 Mexican adults 20-59 years old, of whom $57 \cdot 13 \%$ were in the 20-39 age group. A higher proportion of men had smoked
Table 1. Food group classifications

\begin{tabular}{|c|c|}
\hline Food group & Foods and beverages included \\
\hline $\begin{array}{l}\text { Sugar-sweetened dairy } \\
\text { beverages }\end{array}$ & $\begin{array}{l}\text { Milk with added sugars, drinkable } \\
\text { yoghurt and Mexican milk-based } \\
\text { beverages (atoles) (homemade or } \\
\text { commercially prepared) }\end{array}$ \\
\hline $\begin{array}{l}\text { Non-sugar-sweetened dairy } \\
\text { beverages }\end{array}$ & $\begin{array}{l}\text { Milk without added sugars (all types: } \\
\text { whole, semi-skim, skim, lactose-free, } \\
\text { etc.) }\end{array}$ \\
\hline $\begin{array}{l}\text { Sugar-sweetened non-dairy } \\
\text { beverages }\end{array}$ & $\begin{array}{l}\text { Water-based atole, soft drinks, natural } \\
\text { and industrialised fruit juices and } \\
\text { sugar-sweetened natural fruit water }\end{array}$ \\
\hline $\begin{array}{l}\text { Non-sugar-sweetened } \\
\text { non-dairy beverages }\end{array}$ & $\begin{array}{l}\text { Water, light soft drinks, } \\
\text { non-sugar-sweetened natural fruit } \\
\text { water and low-calorie beverages }\end{array}$ \\
\hline Fruits & $\begin{array}{l}\text { Strawberry, jicama, papaya, pineapple, } \\
\text { grapefruit, grape, orange, mandarin } \\
\text { orange, apple, pear, cantaloupe, } \\
\text { watermelon, peach, banana, guava } \\
\text { and mango }\end{array}$ \\
\hline Vegetables & $\begin{array}{l}\text { Cucumber, carrot, tomato, chayote, } \\
\text { leafy greens (chard, spinach, } \\
\text { pigweed), cabbage, avocado, lettuce, } \\
\text { broccoli, carrot, maize, zucchini, chilli } \\
\text { pepper (all types) and onion (other } \\
\text { root vegetables were not included) }\end{array}$ \\
\hline $\begin{array}{l}\text { Dairy products } \\
\text { (non-beverage) }\end{array}$ & $\begin{array}{l}\text { Yoghurt (natural, light or fruit-added) } \\
\text { and cheese of all types }\end{array}$ \\
\hline Legumes & $\begin{array}{l}\text { Beans, lentils, chickpeas, broad beans } \\
\text { and string beans (fresh or canned) }\end{array}$ \\
\hline Cereal-based salty dishes & Rice and pasta \\
\hline Corn-based savory dishes & $\begin{array}{l}\text { Corn-based fried and non-fried Mexican } \\
\text { dishes: sopes, quesadillas, } \\
\text { enchiladas, tacos, tlacoyos, gorditas, } \\
\text { pozole, tamales }\end{array}$ \\
\hline Fast food & $\begin{array}{l}\text { Hamburgers, pizza, hot dogs and } \\
\text { sandwiches }\end{array}$ \\
\hline Eggs & $\begin{array}{l}\text { Boiled eggs, fried eggs and } \\
\text { egg-battered vegetables }\end{array}$ \\
\hline Meat, poultry and fish & Poultry, beef, pork, fish and shellfish \\
\hline Processed meats & Processed ham and sausage \\
\hline Pastries and cookies & $\begin{array}{l}\text { Donuts, cakes, pies, cupcakes, cookies } \\
\text { and pastries }\end{array}$ \\
\hline Candies & $\begin{array}{l}\text { Chocolate, candies, marshmallows and } \\
\text { Iollipops }\end{array}$ \\
\hline Desserts & $\begin{array}{l}\text { Fried plantains, gelatins, ice cream, } \\
\text { frozen juice bars, sorbet, dried fruit } \\
\text { and candied fruit }\end{array}$ \\
\hline Salty snacks & Popcorn and chips (all kinds) \\
\hline Nuts and seeds & $\begin{array}{l}\text { Peanuts, tree nuts and fried broad } \\
\text { beans }\end{array}$ \\
\hline Added fats & $\begin{array}{l}\text { Butter, lard, vegetable oil, margarine, } \\
\text { mayonnaise and cream }\end{array}$ \\
\hline Tortillas & $\begin{array}{l}\text { Corn tortilla (homemade or } \\
\text { commercially prepared). The corn } \\
\text { tortilla is a staple food in traditional } \\
\text { Mexican cooking; it is a thin, circular } \\
\text { unleavened flatbread made of corn } \\
\text { flour }\end{array}$ \\
\hline Soups & $\begin{array}{l}\text { Instant soups, vegetable soups and } \\
\text { broths (beef, chicken and vegetable) }\end{array}$ \\
\hline Ready-to-eat cereals & $\begin{array}{l}\text { Ready-to-eat cereals (all kinds): basic, } \\
\text { fibre-added, multi-ingredient, } \\
\text { sugar-sweetened or flavoured }\end{array}$ \\
\hline Breads & $\begin{array}{l}\text { White bread, whole-wheat bread and } \\
\text { crackers }\end{array}$ \\
\hline Potatoes & Boiled or fried potatoes \\
\hline $\begin{array}{l}\text { Alcoholic beverages, coffee } \\
\text { and tea }\end{array}$ & $\begin{array}{l}\text { Coffee, tea and alcoholic beverages } \\
\text { (all kinds) }\end{array}$ \\
\hline
\end{tabular}


Table 2. Descriptive characteristics of Mexican adult study participants, by sex

\begin{tabular}{|c|c|c|c|c|c|c|c|c|c|c|}
\hline \multirow[b]{2}{*}{ Characteristic } & \multicolumn{4}{|c|}{ Male } & \multicolumn{4}{|c|}{ Female } & \multirow[b]{2}{*}{ National \% } & \multirow[b]{2}{*}{$95 \% \mathrm{Cl}$} \\
\hline & $n$ & $N^{a}$ & $\%$ & $95 \% \mathrm{Cl}$ & $n$ & $N^{a}$ & $\%$ & $95 \% \mathrm{Cl}$ & & \\
\hline \multicolumn{11}{|l|}{ Age group (years) } \\
\hline $20-39$ & 848 & 16660.06 & 58.74 & $55 \cdot 05,62 \cdot 34$ & 1728 & 17188.69 & $55 \cdot 64$ & $52 \cdot 68,58 \cdot 57$ & $57 \cdot 13$ & $54.58,59 \cdot 64$ \\
\hline $40-59$ & 888 & 11701.44 & $41 \cdot 26$ & $37.66,44.95$ & 1612 & 13701.52 & $44 \cdot 36$ & $41 \cdot 43,47 \cdot 32$ & $42 \cdot 87$ & $40 \cdot 36,45 \cdot 42$ \\
\hline \multicolumn{11}{|l|}{ Area type } \\
\hline Rural & 928 & $7659 \cdot 29$ & $27 \cdot 01$ & $23 \cdot 45,30 \cdot 88$ & 1665 & 7348.91 & 23.79 & $20 \cdot 64,27 \cdot 25$ & $25 \cdot 33$ & $22 \cdot 39,28 \cdot 51$ \\
\hline Urban & 808 & $20702 \cdot 21$ & 72.99 & $69 \cdot 12,76 \cdot 55$ & 1675 & 23541.30 & $76 \cdot 21$ & $72 \cdot 75,79 \cdot 36$ & 74.67 & $71 \cdot 49,77 \cdot 61$ \\
\hline \multicolumn{11}{|l|}{ Geographical region } \\
\hline North & 379 & 5907.33 & $20 \cdot 83$ & $17 \cdot 49,24 \cdot 62$ & 736 & $6510 \cdot 70$ & 21.08 & $16 \cdot 99,25 \cdot 84$ & 20.96 & $17 \cdot 86,24 \cdot 44$ \\
\hline Centre & 576 & 9734.7 & 34.32 & $29.99,38.93$ & 1135 & 10285.98 & 33.30 & $29 \cdot 42,37 \cdot 41$ & 33.79 & $30 \cdot 29,37 \cdot 47$ \\
\hline Mexico City & 182 & 4511.95 & $15 \cdot 91$ & $12 \cdot 53,20 \cdot 00$ & 375 & 5040.54 & $16 \cdot 32$ & $12 \cdot 21,21 \cdot 47$ & $16 \cdot 12$ & $13 \cdot 10,19 \cdot 69$ \\
\hline South & 599 & 8207.52 & 28.94 & $25 \cdot 02,33 \cdot 2$ & 1094 & 9052.99 & $29 \cdot 31$ & $25 \cdot 08,33.92$ & $29 \cdot 13$ & $25 \cdot 56,32 \cdot 98$ \\
\hline \multicolumn{11}{|l|}{ Well-being index } \\
\hline Tertile 1 & 877 & $9762 \cdot 14$ & 34.42 & $30 \cdot 36,38 \cdot 72$ & 1557 & 9935.07 & $32 \cdot 16$ & $28 \cdot 66,35 \cdot 88$ & 33.24 & $30 \cdot 19,36 \cdot 45$ \\
\hline Tertile 2 & 521 & 9577.38 & 33.77 & $30.54,37 \cdot 16$ & 1119 & 10168.98 & 32.92 & $29 \cdot 62,36 \cdot 4$ & 33.33 & $30 \cdot 9,35 \cdot 85$ \\
\hline Tertile 3 & 338 & 9021.99 & 31.81 & $27.99,35.9$ & 664 & $10786 \cdot 16$ & 34.92 & $30.41,39.71$ & 33.43 & $30 \cdot 37,36 \cdot 64$ \\
\hline \multicolumn{11}{|l|}{ Tobacco use (lifelong) } \\
\hline$>100$ cigarettes & 762 & $13648 \cdot 72$ & 48.76 & $45 \cdot 06,52 \cdot 48$ & 359 & 4562.53 & $14 \cdot 87$ & $12 \cdot 50,17.59$ & 31.03 & $28 \cdot 51,33 \cdot 68$ \\
\hline$\leq 100$ cigarettes & 483 & 7458.79 & $26 \cdot 65$ & $23 \cdot 76,29 \cdot 75$ & 815 & 9171.53 & 29.88 & $26 \cdot 33,33 \cdot 70$ & 28.34 & $25 \cdot 94,30 \cdot 87$ \\
\hline No cigarettes & 473 & $6882 \cdot 29$ & 24.59 & $21 \cdot 39,28.09$ & 2144 & $16957 \cdot 25$ & $55 \cdot 25$ & $51 \cdot 08,59 \cdot 35$ & $40 \cdot 63$ & $37 \cdot 66,43 \cdot 66$ \\
\hline BMI $\left(\mathrm{kg} / \mathrm{m}^{2}\right)^{\mathrm{b}}$ & 1709 & 27770.66 & $27 \cdot 74$ & $27 \cdot 33,28 \cdot 15$ & 3295 & $30400 \cdot 02$ & 28.90 & $28 \cdot 53,29 \cdot 26$ & $28 \cdot 34$ & $28 \cdot 05,28 \cdot 64$ \\
\hline \multicolumn{11}{|l|}{ BMI category } \\
\hline Normal & 498 & $8386 \cdot 10$ & $30 \cdot 20$ & $26 \cdot 81,33 \cdot 81$ & 747 & 7693.31 & $25 \cdot 31$ & $22 \cdot 73,28 \cdot 07$ & $27 \cdot 64$ & $25 \cdot 54,29 \cdot 85$ \\
\hline Overweight & 709 & $11636 \cdot 25$ & 41.90 & $38 \cdot 13,45 \cdot 77$ & 1209 & $10810 \cdot 25$ & 35.56 & $32 \cdot 65,38.58$ & 38.59 & $36 \cdot 2,41 \cdot 03$ \\
\hline Obesity & 502 & $7748 \cdot 31$ & $27 \cdot 90$ & $24.58,31.48$ & 1339 & $11896 \cdot 46$ & $39 \cdot 13$ & $36 \cdot 10,42 \cdot 26$ & 33.77 & $31 \cdot 33,36 \cdot 3$ \\
\hline Hypertension (yes) & 145 & $2154 \cdot 65$ & 7.69 & $5 \cdot 90,9.97$ & 413 & 3801.92 & $12 \cdot 37$ & $10 \cdot 38,14 \cdot 68$ & $10 \cdot 14$ & $8 \cdot 68,11 \cdot 82$ \\
\hline \multicolumn{11}{|l|}{ Physical activity level } \\
\hline Inactive & 176 & 3211.66 & $11 \cdot 71$ & $9 \cdot 45,14.42$ & 492 & 4123.02 & $13 \cdot 81$ & $11.53,16.45$ & $12 \cdot 80$ & $11 \cdot 06,14 \cdot 78$ \\
\hline Moderately active & 128 & 1992.58 & $7 \cdot 27$ & $5 \cdot 04,10 \cdot 36$ & 362 & $3490 \cdot 50$ & 11.69 & $9.94,13.69$ & $9 \cdot 57$ & $8 \cdot 22,11 \cdot 12$ \\
\hline Active & 1380 & $22219 \cdot 67$ & 81.02 & $77 \cdot 29,84 \cdot 27$ & 2381 & 22251.03 & 74.51 & $71 \cdot 52,77 \cdot 28$ & $77 \cdot 63$ & $75 \cdot 38,79 \cdot 73$ \\
\hline \multicolumn{11}{|l|}{ Sleep duration (hours) } \\
\hline $7-9$ & 1198 & 18854.29 & 66.48 & $62 \cdot 55,70 \cdot 19$ & 2410 & $21096 \cdot 55$ & $68 \cdot 30$ & $65 \cdot 33,71 \cdot 11$ & 67.43 & $64 \cdot 96,69 \cdot 79$ \\
\hline$<7$ & 453 & $8005 \cdot 46$ & $28 \cdot 23$ & $25 \cdot 02,31 \cdot 66$ & 704 & 7505.72 & $24 \cdot 30$ & $22 \cdot 10,26 \cdot 64$ & $26 \cdot 18$ & $24 \cdot 14,28 \cdot 32$ \\
\hline$>9$ & 85 & $1501 \cdot 76$ & $5 \cdot 30$ & $3 \cdot 71,7 \cdot 51$ & 226 & 2287.93 & $7 \cdot 41$ & $5 \cdot 48,9.94$ & $6 \cdot 40$ & $5 \cdot 10,8 \cdot 00$ \\
\hline Insomnia (yes) & 223 & $3592 \cdot 26$ & $12 \cdot 67$ & $10 \cdot 51,15 \cdot 19$ & 692 & 6224.75 & $20 \cdot 15$ & $17 \cdot 74,22 \cdot 80$ & $16 \cdot 57$ & $14.94,18 \cdot 33$ \\
\hline Daytime symptoms (yes) & 238 & $4100 \cdot 08$ & 14.46 & $11.99,17.33$ & 747 & $7441 \cdot 65$ & 24.09 & $21 \cdot 22,27 \cdot 21$ & 19.48 & $17 \cdot 53,21 \cdot 59$ \\
\hline Obstructive sleep apnoea (yes) & 438 & 7244.71 & 25.54 & $22 \cdot 50,28 \cdot 85$ & 853 & $8290 \cdot 63$ & $26 \cdot 84$ & $23 \cdot 7,30 \cdot 23$ & $26 \cdot 22$ & $23 \cdot 88,28 \cdot 70$ \\
\hline
\end{tabular}

${ }^{a} N$ (thousands) = Expansion of the sample expressed per 1000 individuals, considering survey sample weights.

${ }^{\mathrm{b}}$ Mean and $95 \% \mathrm{Cl}$.

$>100$ cigarettes in their lifetime, as compared with women (48.76\%; $95 \%$ CI 45.06, $52 \cdot 48$ vs. $14 \cdot 87 \%$; $95 \%$ CI $12 \cdot 50$, 17.59). Conversely, women showed a greater prevalence of obesity, as well as hypertension, insomnia and daytime symptoms. Insomnia was reported by $16.57 \%$ of the study population, and daytime symptoms by $19 \cdot 48 \%$. OSA risk was identified in $26.22 \%$ of the population (Table 2).

\section{Dietary patterns}

We identified three dietary patterns: traditional, industrialised and mixed. The traditional pattern yielded the highest mean intake of tortilla $(42.75 \%)$ and legumes $(4.42 \%)$. The industrialised pattern yielded the highest intake of sugar-sweetened non-dairy beverages $(11.01 \%)$, alcoholic beverages, coffee or tea $(8.45 \%)$, bakery products and cookies $(7.37 \%)$, fast foods $(5.79 \%)$, breads $(5.58 \%)$ and candies $(1.32 \%)$. The mixed pattern yielded the highest intakes of fruits $(9.48 \%)$, meat and poultry $(8.51 \%)$, vegetables $(6 \cdot 19 \%)$, non-sugarsweetened dairy beverages (4.00 \%), sugar-sweetened dairy beverages $(3.71 \%)$ and cereal-based salty recipes $(3.34 \%)$ (Table 3 ).
Table 4 shows that the traditional pattern had the lowest median protein intake. The industrialised pattern had the highest median intake of lipids, as well as of all specific varieties of fats and sugars, and the lowest median fibre intake. The mixed pattern showed the lowest median caffeine intake.

Table 5 shows the distribution of participant characteristics by dietary pattern. Adults with the industrialised dietary pattern exhibited the highest energy intake and included the highest proportion of younger adults (aged 20-39), smokers $(>100$ cigarettes) and individuals at high risk for OSA, while showing the lowest proportion of 'active' individuals by physical activity level. Adults with the traditional pattern included the highest proportion of individuals living in rural areas and in southern Mexico, and of individuals in WBI tertile 1 (low). A higher proportion of adults with the traditional pattern reported sleeping 7-9 h/d and suffering less daytime symptoms, compared with the industrialised pattern.

\section{Associations between dietary patterns and sleep disorders}

Table 6 describes the associations between dietary patterns and insomnia, daytime symptoms, $<7 \mathrm{~h}$ sleep duration and high risk 
Table 3. Percentage of energy intake contribution of each food group, by dietary pattern

\begin{tabular}{|c|c|c|c|c|c|c|}
\hline \multirow[b]{2}{*}{ Food group } & \multicolumn{2}{|c|}{$\begin{array}{l}\text { Traditional dietary pattern } \\
n 1969, N 16847 \cdot 7\end{array}$} & \multicolumn{2}{|c|}{$\begin{array}{c}\text { Industrialised dietary } \\
\text { pattern } n 1683, N 24334.2\end{array}$} & \multicolumn{2}{|c|}{$\begin{array}{l}\text { Mixed dietary pattern } n 1424 \text {, } \\
\qquad N 18069.8\end{array}$} \\
\hline & Mean & $95 \% \mathrm{Cl}$ & Mean & $95 \% \mathrm{Cl}$ & Mean & $95 \% \mathrm{Cl}$ \\
\hline Tortilla $^{a}$ & $42 \cdot 75^{\star, \star \star}$ & $41 \cdot 34,44 \cdot 17$ & $16 \cdot 53$ & $15 \cdot 71,17 \cdot 35$ & $17 \cdot 89$ & $16 \cdot 81,18.97$ \\
\hline Sugar-sweetened non-dairy beverages & $6 \cdot 52$ & $6.01,7.03$ & $11 \cdot 01^{*}$ & $10 \cdot 26,11 \cdot 76$ & $5 \cdot 27^{\star \star, \star \star \star}$ & $4 \cdot 78,5 \cdot 77$ \\
\hline Corn-based savory dishes & $7 \cdot 00$ & $6 \cdot 09,7.92$ & $7 \cdot 45$ & $6 \cdot 69,8 \cdot 21$ & $4.91^{\star *, * \star *}$ & $4 \cdot 27,5.55$ \\
\hline Meat, poultry and fish & 3.91 & $3.54,4.27$ & $7 \cdot 00^{*}$ & $6.48,7.52$ & $8 \cdot 51^{\star *, \star \star \star}$ & $7 \cdot 86,9 \cdot 17$ \\
\hline Fruits & 4.03 & $3 \cdot 69,4.38$ & 3.79 & $3.53,4.06$ & $9 \cdot 48^{\star *, \star \star *}$ & $8.93,10.03$ \\
\hline Pastries and cookies & 4.54 & $4.05,5.03$ & $7 \cdot 37^{*}$ & $6.77,7.98$ & $4 \cdot 93^{\star \star \star}$ & $4.44,5.43$ \\
\hline Alcoholic beverages, coffee and tea & 4.88 & $4 \cdot 28,5 \cdot 47$ & $8 \cdot 45^{\star}$ & $7.47,9.43$ & $3 \cdot 22^{\star \star, \star \star \star}$ & $2 \cdot 76,3 \cdot 69$ \\
\hline Eggs & 3.85 & $3 \cdot 43,4 \cdot 26$ & $4 \cdot 07$ & $3 \cdot 72,4.42$ & 3.44 & $3 \cdot 13,3.75$ \\
\hline Breads & $2 \cdot 12$ & $1 \cdot 8,2 \cdot 44$ & $5 \cdot 58^{*}$ & $4.86,6 \cdot 29$ & $3 \cdot 62^{* *, \star \star *}$ & $3.08,4 \cdot 16$ \\
\hline Vegetables & $2 \cdot 30$ & $2 \cdot 12,2 \cdot 49$ & 2.42 & $2 \cdot 22,2 \cdot 62$ & $6 \cdot 19^{\star \star \star \star \star \star}$ & $5 \cdot 65,6 \cdot 73$ \\
\hline Fast food & 1.04 & $0.85,1.23$ & $5 \cdot 79^{\star}$ & $5 \cdot 25,6 \cdot 32$ & $2 \cdot 79^{\star *, \star \star \star}$ & $2 \cdot 36,3.22$ \\
\hline Dairy products (non-beverage) & 1.87 & $1.59,2 \cdot 15$ & $2 \cdot 65^{\star}$ & $2.31,2.99$ & $4 \cdot 87^{\star *, \star \star \star}$ & $4 \cdot 42,5 \cdot 33$ \\
\hline Legumes & 4.42 & $4.02,4.82$ & $2 \cdot 08^{*}$ & $1 \cdot 89,2 \cdot 27$ & $2 \cdot 64^{\star *, \star \star \star}$ & $2 \cdot 41,2 \cdot 87$ \\
\hline Cereal-based salty dishes & 2.65 & $2.39,2.9$ & $2 \cdot 21^{*}$ & $2.04,2.38$ & $3 \cdot 34^{\star \star, \star \star \star}$ & $3.08,3.61$ \\
\hline Non-sugar-sweetened dairy beverages & 1.44 & $1.14,1.73$ & 1.89 & $1 \cdot 63,2 \cdot 14$ & $4 \cdot 00^{\star \star, \star \star \star}$ & $3.39,4.62$ \\
\hline Sugar-sweetened dairy beverages & $1 \cdot 27$ & $0.97,1.57$ & 1.66 & $1 \cdot 38,1.93$ & $3.71^{\star *, \star \star *}$ & $3 \cdot 04,4 \cdot 38$ \\
\hline Processed meats & 0.96 & $0.82,1.09$ & $1 \cdot 82^{*}$ & $1 \cdot 63,2 \cdot 02$ & $1 \cdot 32^{* *, \star * *}$ & $1.09,1.55$ \\
\hline Desserts & 0.52 & $0.43,0.62$ & $1 \cdot 13^{*}$ & $0.99,1 \cdot 27$ & $1.91^{\star *, * * *}$ & $1 \cdot 68,2 \cdot 13$ \\
\hline Potatoes & 1.01 & $0 \cdot 85,1 \cdot 17$ & 0.98 & $0 \cdot 85,1 \cdot 11$ & $1 \cdot 21$ & $1.04,1.39$ \\
\hline Non-sugar-sweetened non-dairy beverages & 0.66 & $0.53,0.79$ & 0.69 & $0.6,0.77$ & $1.44^{\star *, * \star *}$ & $1.21,1.67$ \\
\hline Salty snacks & 0.47 & $0.34,0.59$ & $1.59^{*}$ & $1 \cdot 38,1 \cdot 8$ & $0.58^{\star * \star}$ & $0.45,0.72$ \\
\hline Soups & 0.57 & $0.5,0.64$ & 0.64 & $0.58,0.7$ & $1.53^{\star \star, \star \star \star}$ & $1 \cdot 36,1 \cdot 7$ \\
\hline Added fats & $0.56^{\star, \star \star}$ & $0.31,0.81$ & 0.99 & $0 \cdot 84,1 \cdot 14$ & 0.93 & $0.76,1 \cdot 1$ \\
\hline Candies & 0.26 & $0.19,0.33$ & $1 \cdot 32^{*}$ & $1 \cdot 15,1 \cdot 48$ & $0.58^{* *, * * *}$ & $0.48,0.67$ \\
\hline Ready-to-eat cereals & 0.12 & $0.09,0.16$ & $0.31^{*}$ & $0.24,0.38$ & $1 \cdot 13^{\star *, \star \star *}$ & $0.95,1.32$ \\
\hline Nuts and seeds & $0.29^{\star, \star \star}$ & $0.21,0.38$ & 0.58 & $0.44,0.73$ & 0.53 & $0.37,0.69$ \\
\hline
\end{tabular}

a The corn tortilla is a staple food in traditional Mexican cooking; it is a thin, circular unleavened flatbread made of corn flour.

* Significant difference between the traditional and industrialised patterns $(P<0.017)$.

** Significant difference between the traditional and mixed patterns $(P<0.017)$.

*** Significant difference between the industrialised and mixed patterns $(P<0.017)$.

for OSA (all at the national level and stratified by sex). Adults with the industrialised dietary pattern had higher odds of experiencing daytime symptoms than those with the traditional pattern (OR 1.49; $P=0 \cdot 007)$, mainly due to an outsized effect in women. Furthermore, adults with the industrialised dietary pattern showed significantly higher odds of being at high risk for OSA (OR 1.63; $P=0.001)$ than those with the traditional dietary pattern, driven by a significant association in men. For women, the industrialised dietary pattern increased odds for sleep duration $<7 \mathrm{~h}$ (OR $1 \cdot 63 ; P=0 \cdot 04)$. Insomnia was not associated with any dietary pattern in this sample. Complete logistic regression models are given in Supplementary Table S1 of Supplementary material.

In the present study sample, 131 subjects reported the use of sedative medications $(2 \cdot 62 \%$; $95 \%$ CI $1.98,3.44)$; logistic regression models were run excluding these participants and subsequent estimations remained unchanged (data not shown).

Table 4. Nutritional characteristics, by dietary pattern

\begin{tabular}{|c|c|c|c|c|c|c|}
\hline \multirow[b]{2}{*}{ Energy and nutrients } & \multicolumn{2}{|c|}{ Traditional dietary pattern } & \multicolumn{2}{|c|}{ Industrialised dietary pattern } & \multicolumn{2}{|c|}{ Mixed dietary pattern } \\
\hline & Median & $\mathrm{p} 25, \mathrm{p} 75$ & Median & p25, p75 & Median & p25, p75 \\
\hline Energy (kcal/d) & $1802 \cdot 89$ & $1355 \cdot 42,2391.62$ & $2115 \cdot 07^{*, \star * *}$ & $1618 \cdot 74,2806 \cdot 90$ & $1681 \cdot 58$ & $1293 \cdot 82,2220 \cdot 36$ \\
\hline Carbohydrates (g/d) & 300.93 & $227 \cdot 74,380 \cdot 08$ & 298.05 & 223.46, 389.89 & $237.95^{\star *, \star \star *}$ & $188 \cdot 42,313 \cdot 68$ \\
\hline Lipids (g/d) & 44.54 & $31 \cdot 78,63 \cdot 44$ & $66 \cdot 66^{*}$ & $49 \cdot 40,90 \cdot 37$ & $55 \cdot 60^{\star \star, \star \star \star}$ & $38 \cdot 88,78 \cdot 29$ \\
\hline Proteins $(\mathrm{g} / \mathrm{d})$ & $55 \cdot 59^{\star, * \star}$ & $40 \cdot 19,74 \cdot 20$ & $65 \cdot 57$ & $50 \cdot 18,88 \cdot 38$ & 63.09 & $44.52,81 \cdot 81$ \\
\hline Fibers (g/d) & $30 \cdot 61$ & $22 \cdot 04,40 \cdot 42$ & $22 \cdot 25^{*}$ & $15 \cdot 83,30 \cdot 38$ & $25 \cdot 91^{\star *, \star \star \star}$ & $18 \cdot 37,34 \cdot 20$ \\
\hline Sugars (g/d) & $69 \cdot 70$ & $45 \cdot 62,102 \cdot 49$ & $121 \cdot 68^{*}$ & $81.91,184.43$ & $95 \cdot 33^{\star *, * \star *}$ & $68.79,129.95$ \\
\hline Caffeine (mg/d) & $85 \cdot 25$ & $15 \cdot 86,221 \cdot 80$ & $101 \cdot 73$ & $38 \cdot 43,225 \cdot 32$ & $33 \cdot 51^{\star *, \star \star \star}$ & $7 \cdot 80,148 \cdot 15$ \\
\hline Alcohol (g/d) & 0.00 & $0.00,0.002$ & 0.002 & $0.00,17.48$ & 0.00 & $0.00,0.01$ \\
\hline Saturated fats $(\mathrm{g} / \mathrm{d})$ & 14.69 & $9 \cdot 29,20 \cdot 91$ & $24 \cdot 08^{*}$ & $17 \cdot 44,33.40$ & $20 \cdot 31^{* *, * \star *}$ & $13 \cdot 52,29 \cdot 71$ \\
\hline Monounsaturated fats $(\mathrm{g} / \mathrm{d})$ & $13 \cdot 81$ & $9.54,19.63$ & $22 \cdot 57^{*}$ & $16 \cdot 45,29 \cdot 00$ & $18 \cdot 09^{\star \star, \star \star \star}$ & $12 \cdot 01,26 \cdot 74$ \\
\hline Polyunsaturated fats (g/d) & $12 \cdot 87$ & $8 \cdot 75,18 \cdot 41$ & $15 \cdot 68^{*}$ & $11 \cdot 30,23 \cdot 04$ & $11 \cdot 62^{\star \star, \star \star \star}$ & $7 \cdot 98,17 \cdot 33$ \\
\hline Trans fats $(\mathrm{g} / \mathrm{d})$ & 0.52 & $0.20,1.32$ & $1 \cdot 14^{*}$ & $0.57,2 \cdot 24$ & $1 \cdot 13^{\star \star, \star \star \star}$ & $0.47,2 \cdot 71$ \\
\hline
\end{tabular}

* Significant difference between the traditional and industrialised patterns $(P<0.017)$.

${ }^{*}$ Significant difference between the traditional and mixed patterns $(P<0.017)$.

*** Significant difference between the industrialised and mixed patterns $(P<0.017)$. 
Table 5. Descriptive characteristics of Mexican adult study participants, by dietary pattern

\begin{tabular}{|c|c|c|c|c|c|c|c|c|c|}
\hline \multirow[b]{2}{*}{ Characteristic } & \multicolumn{3}{|c|}{ Traditional dietary pattern } & \multicolumn{3}{|c|}{ Industrialised dietary pattern } & \multicolumn{3}{|c|}{ Mixed dietary pattern } \\
\hline & $n$ & $\%$ & $95 \% \mathrm{Cl}$ & $n$ & $\%$ & $95 \% \mathrm{Cl}$ & $n$ & $\%$ & $95 \% \mathrm{Cl}$ \\
\hline \multicolumn{10}{|l|}{ Sex } \\
\hline Male & 745 & $52 \cdot 10$ & $48 \cdot 44,55 \cdot 74$ & 688 & $56 \cdot 11$ & $52 \cdot 54,59 \cdot 61$ & 303 & $32 \cdot 82^{\star *, * * *}$ & $28.13,37.89$ \\
\hline Female & 1224 & $47 \cdot 90$ & $44.26,51 \cdot 56$ & 995 & $43 \cdot 89$ & $40 \cdot 39,47 \cdot 46$ & 1121 & $67 \cdot 18^{\star, \star \star \star}$ & $62 \cdot 11,71 \cdot 87$ \\
\hline \multicolumn{10}{|l|}{ Age group (years) } \\
\hline 20-39 & 921 & $50 \cdot 57$ & $46 \cdot 53,54 \cdot 6$ & 993 & $64 \cdot 48^{*, * \star *}$ & $60 \cdot 67,68 \cdot 12$ & 662 & $53 \cdot 33$ & $48.58,58.02$ \\
\hline $40-59$ & 1048 & $49 \cdot 43$ & $45 \cdot 4,53.47$ & 690 & $35 \cdot 52^{*, * \star *}$ & $31 \cdot 88,39 \cdot 33$ & 762 & $46 \cdot 67$ & $41.98,51.42$ \\
\hline \multicolumn{10}{|l|}{ Area type } \\
\hline Rural & 1352 & $44 \cdot 88^{\star, \star \star}$ & $39.42,50.46$ & 682 & $17 \cdot 56$ & $14 \cdot 37,21 \cdot 29$ & 559 & $17 \cdot 56$ & $14.07,21 \cdot 71$ \\
\hline Urban & 617 & $55 \cdot 12^{\star, \star \star \star}$ & $49.54,60.58$ & 1001 & 82.44 & $78 \cdot 71,85 \cdot 63$ & 865 & 82.44 & $78.29,85.93$ \\
\hline \multicolumn{10}{|l|}{ Geographical region } \\
\hline North & 364 & $13 \cdot 82^{*}$ & $10 \cdot 13,18.57$ & 510 & 27.03 & $23 \cdot 28,31 \cdot 13$ & 241 & 19.44 & $13 \cdot 65,26 \cdot 93$ \\
\hline Centre & 652 & $33 \cdot 80$ & $28 \cdot 83,39 \cdot 16$ & 540 & 34.27 & $29 \cdot 8,39 \cdot 05$ & 519 & $33 \cdot 12$ & $28.11,38.55$ \\
\hline Mexico City & 100 & $8.43^{\star, \star \star}$ & $5 \cdot 93,11 \cdot 86$ & 248 & $18 \cdot 80$ & $15 \cdot 43,22 \cdot 72$ & 209 & 19.68 & $14 \cdot 49,26 \cdot 17$ \\
\hline South & 853 & $43 \cdot 95^{*, \star *}$ & $38 \cdot 33,49 \cdot 72$ & 385 & $19 \cdot 90$ & $16 \cdot 31,24 \cdot 04$ & 455 & $27 \cdot 75$ & $22 \cdot 74,33.39$ \\
\hline \multicolumn{10}{|l|}{ Well-being index } \\
\hline Tertile 1 & 1334 & $58 \cdot 05^{*, * \star}$ & $53.02,62.92$ & 616 & $24 \cdot 16$ & $20 \cdot 95,27 \cdot 7$ & 484 & $22 \cdot 34$ & $18 \cdot 77,26 \cdot 37$ \\
\hline Tertile 2 & 496 & $31 \cdot 01$ & $26 \cdot 8,35 \cdot 56$ & 622 & 35.09 & $31 \cdot 2,39 \cdot 19$ & 522 & $33 \cdot 11$ & $28 \cdot 65,37 \cdot 89$ \\
\hline Tertile 3 & 139 & $10 \cdot 94^{*, * *}$ & $8.484,13.99$ & 445 & $40 \cdot 75$ & $36 \cdot 42,45 \cdot 23$ & 418 & 44.55 & $38 \cdot 56,50 \cdot 7$ \\
\hline \multicolumn{10}{|l|}{ Tobacco use (lifetime) } \\
\hline >100 cigarettes & 358 & $25 \cdot 60$ & $22 \cdot 18,29 \cdot 35$ & 519 & $42 \cdot 61^{*, \star \star *}$ & $38 \cdot 52,46 \cdot 8$ & 244 & $20 \cdot 53$ & $16 \cdot 43,25 \cdot 35$ \\
\hline$\leq 100$ cigarettes & 473 & $27 \cdot 06$ & $23.43,31.03$ & 450 & $26 \cdot 36$ & $22 \cdot 87,30 \cdot 18$ & 375 & $32 \cdot 18$ & $27 \cdot 38,37 \cdot 38$ \\
\hline None & 1125 & $47 \cdot 34$ & $42 \cdot 93,51 \cdot 79$ & 700 & $31 \cdot 03^{*, \star \star *}$ & $27 \cdot 44,34 \cdot 87$ & 792 & $47 \cdot 29$ & $41.47,53.19$ \\
\hline $\mathrm{BMI}^{\mathrm{a}}$ & 1947 & $27 \cdot 87^{\star}$ & $27 \cdot 43,28 \cdot 31$ & 1657 & $28 \cdot 74$ & $28 \cdot 23,29 \cdot 25$ & 1400 & $28 \cdot 26$ & $27 \cdot 75,28 \cdot 77$ \\
\hline \multicolumn{10}{|l|}{ BMI category } \\
\hline Normal & 523 & 28.64 & $25 \cdot 07,32 \cdot 5$ & 404 & $25 \cdot 70$ & $22 \cdot 26,29 \cdot 48$ & 318 & $29 \cdot 30$ & $24.94,34.06$ \\
\hline Overweight & 782 & $43 \cdot 16$ & $38.99,47.43$ & 600 & $37 \cdot 21$ & $33 \cdot 32,41 \cdot 27$ & 536 & $36 \cdot 12$ & $31.75,40 \cdot 73$ \\
\hline Obese & 642 & $28 \cdot 20^{*}$ & $24 \cdot 46,32 \cdot 27$ & 653 & 37.09 & $33 \cdot 1,41 \cdot 26$ & 546 & 34.58 & $30 \cdot 65,38 \cdot 74$ \\
\hline Hypertension (yes) & 204 & $9 \cdot 36$ & $7 \cdot 164,12 \cdot 14$ & 177 & $10 \cdot 61$ & $8 \cdot 398,13 \cdot 31$ & 177 & $10 \cdot 24$ & $7.793,13.33$ \\
\hline \multicolumn{10}{|l|}{ Physical activity level } \\
\hline Inactive & 236 & $12 \cdot 36$ & $9 \cdot 54,15 \cdot 86$ & 254 & $16 \cdot 00$ & $13 \cdot 05,19 \cdot 46$ & 178 & $8 \cdot 98^{\star \star \star}$ & $6.956,11.51$ \\
\hline Moderately active & 161 & 7.05 & $5 \cdot 33,9 \cdot 259$ & 180 & $10 \cdot 51$ & $8 \cdot 322,13 \cdot 18$ & 149 & $10 \cdot 67$ & $8 \cdot 394,13.48$ \\
\hline Active & 1508 & $80 \cdot 60$ & $76 \cdot 61,84.05$ & 1204 & $73 \cdot 50^{*, \star \star \star}$ & $69.66,77.01$ & 1049 & $80 \cdot 35$ & $76 \cdot 94,83.36$ \\
\hline \multicolumn{10}{|l|}{ Sleep duration (hours) } \\
\hline $7-9$ & 1472 & $73 \cdot 12^{\star}$ & $69 \cdot 19,76 \cdot 73$ & 1130 & $62 \cdot 52$ & $58 \cdot 76,66 \cdot 13$ & 1006 & $68 \cdot 72$ & $63 \cdot 29,73 \cdot 68$ \\
\hline$<7$ & 377 & $20 \cdot 98^{*}$ & $17 \cdot 86,24 \cdot 48$ & 456 & 31.30 & $27 \cdot 76,35.08$ & 324 & $24 \cdot 13$ & $20 \cdot 28,28 \cdot 44$ \\
\hline$>9$ & 120 & 5.90 & $4.14,8.33$ & 97 & $6 \cdot 18$ & $4.47,8.48$ & 94 & $7 \cdot 15$ & $4.53,11 \cdot 11$ \\
\hline Insomnia (yes) & 292 & $15 \cdot 85$ & $13 \cdot 2,18 \cdot 92$ & 356 & $17 \cdot 26$ & $14 \cdot 78,20 \cdot 06$ & 267 & $16 \cdot 31$ & $13 \cdot 42,19 \cdot 68$ \\
\hline Daytime symptoms (yes) & 337 & $15 \cdot 54^{*}$ & $13.07,18.39$ & 387 & $22 \cdot 66$ & $19 \cdot 45,26 \cdot 22$ & 261 & $18 \cdot 86$ & $15 \cdot 58,22 \cdot 66$ \\
\hline Obstructive sleep apnoea (yes) & 445 & $21 \cdot 68$ & $18 \cdot 27,25 \cdot 53$ & 496 & $30 \cdot 65^{\star, \star \star \star}$ & $27 \cdot 27,34 \cdot 24$ & 350 & 24.49 & $20 \cdot 94,28 \cdot 41$ \\
\hline
\end{tabular}

${ }^{\mathrm{a}}$ Mean and $95 \% \mathrm{Cl}$.

* Significant difference between the traditional and industrialised patterns $(P<0.017)$.

** Significant difference between the traditional and mixed patterns $(P<0.017)$.

${ }^{* * *}$ Significant difference between the industrialised and mixed patterns $(P<0.017)$.

\section{Discussion}

We identified three dietary patterns in our study population: industrialised, traditional and mixed. The industrialised pattern was defined by a high intake of food groups containing refined sugars, saturated fats and stimulant components such as caffeine and alcohol. The present results show that Mexican adults with an industrialised dietary pattern are significantly more likely to experience daytime symptoms and OSA than those with a traditional dietary pattern.

Although these associations differed in their level of significance by sex, it is important to note that the direction of the association is the same for each sex. This could be explained knowing that the most frequent sleep disorders in women are mainly daytime sleepiness ${ }^{(52)}$ and insufficient sleep time, while in men, OSA is more frequent ${ }^{(53)}$. The lack of statistical significance for daytime symptoms and sleep duration $(P<$ $0 \cdot 05$ ) in men could be due in part to our sample size.

Notably, the industrialised pattern remained the pattern most associated with sleep disorders in both sexes.

Similar results have been obtained in other contexts. For example, a study in China revealed that the traditional dietary pattern - characterised by high intakes of wheat and other staple foods - was associated with a decreased prevalence of daytime symptoms (OR 0.94; $95 \%$ CI 0.89, 0.99; $P=0.004)$ and sleep disorders ${ }^{(28)}$. Likewise, when analysing the association between the Mediterranean diet and sleep quality, St-Onge et al. found that increased caloric intake was linked to daytime sleepiness. The high caloric intake observed by these authors may have been related to the consumption of more industrialised foods, in contrast with the Mediterranean diet ${ }^{(54)}$. Although the so-called traditional dietary patterns in these studies are not comparable in cultural terms, they share certain characteristics such as lower meat consumption and higher cereal consumption, as well as lower energy intake as compared with their counterparts. 
Table 6. Associations among dietary patterns and sleep disorders in Mexican adult study participants (Odds ratio and $95 \%$ confidence interval)

\begin{tabular}{|c|c|c|c|c|c|c|c|c|c|}
\hline & \multicolumn{3}{|c|}{ Males } & \multicolumn{3}{|c|}{ Females } & \multicolumn{3}{|c|}{ National } \\
\hline & OR & $95 \% \mathrm{Cl}$ & $P$-value & OR & $95 \% \mathrm{Cl}$ & $P$-value & OR & $95 \% \mathrm{Cl}$ & $P$-value \\
\hline \multicolumn{10}{|l|}{ Insomnia ${ }^{a}$} \\
\hline \multicolumn{10}{|l|}{ Dietary pattern } \\
\hline Traditional & 1.00 & & & & & & 1.00 & & \\
\hline Industrialised & 1.05 & $0.58,1.90$ & 0.878 & $1 \cdot 11$ & $0.75,1.63$ & 0.601 & 1.08 & $0.77,1.53$ & 0.654 \\
\hline Mixed & 0.92 & $0.52,1.62$ & 0.768 & 0.87 & $0.60,1.24$ & 0.432 & 0.87 & $0.63,1.19$ & 0.387 \\
\hline \multicolumn{10}{|l|}{ Sex } \\
\hline Male & & & & & & & 1.00 & & \\
\hline Female & & & & & & & 1.65 & $1 \cdot 18,2 \cdot 3$ & 0.003 \\
\hline \multicolumn{10}{|l|}{ Daytime symptoms ${ }^{a}$} \\
\hline \multicolumn{10}{|l|}{ Dietary pattern } \\
\hline Traditional & 1.00 & & & 1.00 & & & 1.00 & & \\
\hline Industrialised & 1.42 & $0.82,2.46$ & 0.208 & 1.51 & $1 \cdot 04,2 \cdot 19$ & 0.032 & 1.49 & $1.12,1.99$ & 0.007 \\
\hline Mixed & 1.45 & $0.78,2.70$ & 0.239 & 0.75 & $0.51,1.08$ & 0.121 & 0.93 & $0.66,1 \cdot 3$ & 0.665 \\
\hline \multicolumn{10}{|l|}{ Sex } \\
\hline Male & & & & & & & 1.00 & & \\
\hline Female & & & & & & & $2 \cdot 17$ & $1.51,3.13$ & $<0.0001$ \\
\hline \multicolumn{10}{|c|}{ Sleep duration $<7 \mathrm{~h}^{\mathrm{a}}$} \\
\hline \multicolumn{10}{|l|}{ Dietary pattern } \\
\hline Traditional & 1.00 & & & 1.00 & & & 1.00 & & \\
\hline Industrialised & 1.06 & $0.68,1.67$ & 0.797 & 1.62 & $1.02,2.58$ & 0.040 & 1.32 & $0.96,1.8$ & 0.088 \\
\hline Mixed & 0.76 & $0.441,1.3$ & 0.323 & $1 \cdot 18$ & $0.80,1.76$ & 0.405 & 0.98 & $0.71,1.36$ & 0.906 \\
\hline \multicolumn{10}{|l|}{ Sex } \\
\hline Male & & & & & & & 1.00 & & \\
\hline Female & & & & & & & 0.84 & $0.65,1.08$ & 0.176 \\
\hline \multicolumn{10}{|c|}{ Obstructive sleep apnoea ${ }^{b}$} \\
\hline \multicolumn{10}{|c|}{ Dietary pattern } \\
\hline Traditional & 1.00 & & & 1.00 & & & 1.00 & & \\
\hline Industrialised & $1 \cdot 86$ & $1 \cdot 24,2 \cdot 81$ & 0.003 & 1.41 & $0 \cdot 9,2 \cdot 14$ & 0.113 & 1.63 & $1 \cdot 21,2 \cdot 19$ & 0.001 \\
\hline Mixed & $1 \cdot 31$ & $0.82,2.11$ & 0.253 & 1.00 & $0.65,1.54$ & 0.992 & $1 \cdot 14$ & $0.83,1.55$ & 0.424 \\
\hline \multicolumn{10}{|l|}{ Sex } \\
\hline Male & & & & & & & 1.00 & & \\
\hline Female & & & & & & & 1.25 & $0.93,1.68$ & 0.14 \\
\hline
\end{tabular}

${ }^{\text {a }}$ Adjusted by age, sex, body mass index, rural/urban area type, geographical region, physical activity level, lifetime tobacco use, tertiles of well-being index and total energy intake (kcal).

${ }^{\mathrm{b}}$ Adjusted by age, sex, rural/urban area type, geographical region, physical activity level, lifetime tobacco use, tertiles of well-being index and total energy intake (kcal).

A study in peri-menopausal Mexican women showed that those in the least-healthy dietary pattern quartile of fruit and vegetable intake, as compared with the healthiest, were $21 \%$ more likely to report poor sleep quality at follow-up (95\% CI 1.06, 1.42). Those within the highest quartiles of the 'modern Mexican' pattern (tortillas and sodas, low in fibre and dairy) were $23 \%$ more likely to report poor sleep quality as compared with the lowest quartiles $(95 \% \text { CI } 1 \cdot 06,1 \cdot 43)^{(38)}$.

Finally, a study of female Japanese workers indicated that an increased intake of candies and noodles - as opposed to fish and vegetables - was associated with poor sleep quality. This may have been linked to a high carbohydrate intake ${ }^{(32)}$.

A relationship between high alcohol intake and daytime symptoms was reported by Lohsoonthorn et al., who found that college students consuming $\geq 20$ drinks/month had an OR of 3.10 (95\% CI $1.72,5.59)$ for daytime symptoms ${ }^{(55)}$. Furthermore, when analysing the effect of alcohol on sleep physiology in non-alcoholic adults, Roehrs and Roth observed that alcohol consumption altered sleep stages and reduced rapid eye movement sleep time ${ }^{(56)}$. This may be explained by the effects of alcohol on hormonal functions related to the circadian rhythm; for instance, alcohol intake reduces the number of growth hormones. It has been documented that alcohol consumption affects specific neurotransmitters - e.g., gamma-aminobutyric acid (GABA) and acetylcholine involved in the sleep-wake cycle. Reduced sleep quality hampers daytime alertness. A similar mechanism has been reported regarding the effect of caffeine on sleep disruption and perceived sleep quality, affecting the function of the hormone adenosine in the regulation of sleep-wake cycles ${ }^{(57)}$.

One study in young Mexican-American adults suggested that alcohol-use disorders are significantly associated with poorer sleep quality and that substance-use disorders may affect different aspects of sleep than do anxiety and depressive disorders ${ }^{(58)}$. In addition, a study on Mexican adolescents documented that males with a relatively older age, nonstudents, with more screen time, and having smoked cigarettes at any point, were associated with a later weekday sleep midpoint ${ }^{(37)}$.

In regards to the association between dietary intake and OSA, the present results are compatible with those of Vasquez et al., where patients with OSA and a severe respiratory disturbance index score $(\mathrm{RDI} \geq 50)$ showed higher intakes of cholesterol, saturated fats and trans fats ${ }^{(59)}$. These types of nutrients are key components of the food groups characterising the industrialised dietary pattern. Similar results were reported in Multi-Ethnic Study of Atherosclerosis, which found that adults with moderate to severe OSA had higher 
dietary quality scores. This association was also found with $\mathrm{red} /$ processed meats and whole-grain components scores ${ }^{(60)}$, and $6-9 \%$ of this relationship was explained by a reduction in N3 sleep (slow-wave sleep) duration in individuals with OSA. In the present study, the industrialised pattern was characterised by a higher consumption of processed meats in contrast with the traditional and mixed patterns, as well as showing lower tortilla consumption than the traditional pattern.

Limited information is available on the biological mechanism driving the association between dietary patterns and OSA. Barceló et al. proposed a biological explanation, with the results of their study indicating higher levels of neuropeptide $\mathrm{Y}$ and leptin in OSA patients. Neuropeptide $\mathrm{Y}$ is an appetite-stimulating peptide, and the role of leptin in regulating energy balance is well-established ${ }^{(61)}$. In a laboratory-based randomised crossover study of adults aged 30-45, Shechter reported that subjects with lower N3 sleep increased fat and carbohydrate intake ${ }^{(62)}$. Frank et al. proposed a relationship between sleep quality and sleep disorders, and poor dietary quality ${ }^{(63)}$. In addition, a meta-analysis of randomised clinical trials showed that sleep apnoea was improved with low-energy-intake diets, both as a unique intervention and accompanied by exercise ${ }^{(64)}$. On the other hand, it should be noted that high energy intake, as well as energy-dense or nutrient-dense foods (low in fibre and high in saturated fats and sugars) and poor dietary quality are part of unhealthy routines with multiple components which may impact sleep quality such as sleep hygiene, exercise, obesity, and smoking.

As shown in Table 4, the industrialised pattern had higher intakes of the energy, sugar and fats mentioned earlier, which reinforces the associations previously documented between these consumption habits and sleep disorders.

The present study had some limitations, among them, the cross-sectional design which did not allow the establishment of causality. Furthermore, the obtention of information through self-reporting may have led to over- or underestimation of sleep disorder prevalence, and biased results within certain food group intakes by characteristics such as BMI and sex, given that certain answers may have been perceived as socially desirable $^{(65)}$. In addition, although statistical regression models were adjusted by rural/urban area type, geographical region and physical activity level, residual confusion is possible, and the effects attributed to the industrialised dietary pattern may be further linked to factors such as urban lifestyles in more developed regions, depression or stress.

On the other hand, strengths of the present study include that our sample is nationally representative, and dietary assessment was performed using a food frequency questionnaire validated in both the adolescent and adult Mexican populations which shows an acceptable correlation between consumption, energy and macronutrients intakes estimated by $24 \mathrm{~h}$ recalls and food frequency questionnaire ${ }^{(66)}$. In addition, the dietary pattern approach encompasses the effects of the overall diet, representing a broad picture of food and nutrient consumption and offering a more accurate way of assessing disease risk than do individual food or nutrient intakes ${ }^{(67,68)}$. Furthermore, the association between dietary intake and sleep disorders in the Mexican population serves to generate new hypotheses which demand further research, with the final goal of improving the diet and health of Mexican adults.

\section{Conclusions}

In conclusion, a diet high in sugar-sweetened beverages, fast foods, breads, pastries and cookies, and alcohol, coffee or tea is associated with higher odds for suffering from OSA and daytime symptoms in Mexican adults aged 20-59. It is essential to raise public awareness of the role of diet on sleep quality and health and perform further research, in order to promote evidence-based interventions aimed at achieving healthier diets in the population and preventing the negative effects, including sleep disorders, of unhealthy diets on health.

\section{Supplementary material}

The supplementary material for this article can be found at https://doi.org/10.1017/jns.2021.24.

\section{Acknowledgements}

This work was supported by the Mexican Ministry of Health. The funding body had no role in the design, analysis or drafting of this article.

E. B. G. P. contributed to conceptualisation, formal analysis and drafting. B. M. T. contributed to data cleaning and drafting. S. R. R. contributed to conceptualisation and drafting: reviewing and editing. S. G. Z. contributed to conceptualisation and formal analysis. R. P. P. contributed to data interpretation and drafting. T. S. L. contributed to securing funds and drafting: reviewing and editing.

This study was conducted according to the guidelines set by the Declaration of Helsinki, and all procedures involving human subjects were approved by the Bioethics Board of the National Institute of Public Health of Mexico. Written informed consent was obtained from all participants.

The authors declare that they have no conflicts of interest.

\section{References}

1. Castro-Diehl C, Wood AC, Redline S, et al. (2018) Mediterranean diet pattern and sleep duration and insomnia symptoms in the multi-ethnic study of atherosclerosis. Sleep 41 , zsy158.

2. Hirshkowitz M, Whiton K, Albert SM, et al. (2015) National Sleep Foundation's sleep time duration recommendations: methodology and results summary. Sleep Health 1, 40-43.

3. Wu Y, Zhai L \& Zhang D (2014) Sleep duration and obesity among adults: a meta-analysis of prospective studies. Sleep Med 15, 14561462

4. Stranges S, Dorn JM, Cappuccio FP, et al. (2010) A populationbased study of reduced sleep duration and hypertension: the strongest association may be in premenopausal women. $J$ Hypertens 28, 896-902.

5. Chaput J-P, Després J-P, Bouchard C, et al. (2007) Association of sleep duration with type 2 diabetes and impaired glucose tolerance. Diabetologia 50, 2298-2304.

6. Cappuccio FP, Cooper D, D'elia L, et al. (2011) Sleep duration predicts cardiovascular outcomes: a systematic review and meta-analysis of prospective studies. Eur Heart J 32, 1484-1492. 
7. Cappuccio FP, D’Elia L, Strazzullo P, et al. (2010) Sleep duration and all-cause mortality: a systematic review and meta-analysis of prospective studies. Sleep 33, 585-592.

8. Roth T (2007) Insomnia: definition, prevalence, etiology, and consequences. J Clin Sleep Med 3, 4.

9. Parthasarathy S, Vasquez MM, Halonen M, et al. (2015) Persistent insomnia is associated with mortality risk. $A m J$ Med 128, 268-275.e2.

10. Lallukka T, Podlipskyte A, Sivertsen B, et al. (2016) Insomnia symptoms and mortality: a register-linked study among women and men from Finland, Norway and Lithuania. J Sleep Res 25, 96-103.

11. Sofi F, Cesari F, Casini A, et al. (2014) Insomnia and risk of cardiovascular disease: a meta-analysis. Eur J Prev Cardiol 21, 57-64.

12. Laugsand LE, Strand LB, Vatten LJ, et al. (2014) Insomnia symptoms and risk for unintentional fatal injuries - the HUNT study. Sleep 37, 1777-1786.

13. Tietjens JR, Claman D, Kezirian EJ, et al. (2019) Obstructive sleep apnea in cardiovascular disease: a review of the literature and proposed multidisciplinary clinical management strategy. J Am Heart Assoc 8, e010440.

14. Shahar E, Whitney CW, Redline S, et al. (2001) Sleep-disordered breathing and cardiovascular disease. Am J Respir Crit Care Med $163,19-25$.

15. Slater G \& Steier J (2012) Excessive daytime sleepiness in sleep disorders. J Thorac Dis 4, 9.

16. Gaultney JF (2010) The prevalence of sleep disorders in college students: impact on academic performance. J Am Coll Health 59, 91-97.

17. Haavisto M-L, Porkka-Heiskanen T, Hublin C, et al. (2010) Sleep restriction for the duration of a work week impairs multitasking performance. J Sleep Res 19, 444-454.

18. Uehli K, Mehta AJ, Miedinger D, et al. (2014) Sleep problems and work injuries: a systematic review and meta-analysis. Sleep Med Rev $18,61-73$.

19. Guilleminault C (2001) Excessive daytime sleepiness: a challenge for the practising neurologist. Brain 124, 1482-1491.

20. Moreira P, Santos S, Padrão P, et al. (2010) Food patterns according to sociodemographics, physical activity, sleeping and obesity in Portuguese children. Int J Environ Res Public Health 7, 1121-1138.

21. Chen M-Y, Wang EK \& Jeng Y-J (2006) Adequate sleep among adolescents is positively associated with health status and health-related behaviors. BMC Public Health 6, 59.

22. Weiss A, Xu F, Storfer-Isser A, et al. (2010) The association of sleep duration with adolescents' fat and carbohydrate consumption. Sleep 33, 1201-1209.

23. Westerlund L, Ray C \& Roos E (2009) Associations between sleeping habits and food consumption patterns among 10-11-year-old children in Finland. Br J Nutr 102, 1531-1537.

24. Imaki M, Hatanaka Y, Ogawa Y, et al. (2002) An epidemiological study on relationship between the hours of sleep and life style factors in Japanese factory workers. J Physiol Anthropol Appl Human Sci 21, 115-120.

25. Grandner MA, Kripke DF, Naidoo N, et al. (2010) Relationships among dietary nutrients and subjective sleep, objective sleep, and napping in women. Sleep Med 11, 180-184.

26. Shi Z, McEvoy M, Luu J, et al. (2008) Dietary fat and sleep duration in Chinese men and women. Int J Obes 32, 1835-1840.

27. Peuhkuri K, Sihvola N \& Korpela R (2012) Diet promotes sleep duration and quality. Nutr Res 32, 309-319.

28. Yu C, Shi Z, Lv J, et al. (2017) Dietary patterns and insomnia symptoms in Chinese adults: the China Kadoorie Biobank. Nutrients 9, 232.

29. Kurotani K, Kochi T, Nanri A, et al. (2015) Dietary patterns and sleep symptoms in Japanese workers: the Furukawa Nutrition and Health Study. Sleep Med 16, 298-304.

30. Cao Y, Taylor AW, Wittert G, et al. (2017) Dietary patterns and sleep parameters in a cohort of community dwelling Australian men. Asia Pac J Clin Nutr 26, 1158-1169.

31. Tanaka E, Yatsuya H, Uemura M, et al. (2013) Associations of protein, fat, and carbohydrate intakes with insomnia symptoms among middle-aged Japanese workers. J Epidemiol 23, 132-138.
32. Katagiri R, Asakura K, Kobayashi S, et al. (2014) Low intake of vegetables, high intake of confectionary, and unhealthy eating habits are associated with poor sleep quality among middle-aged female Japanese workers. J Occup Health 56, 359-368.

33. Bouscoulet LT, Vázquez-García JC, Muiño A, et al. (2008) Prevalence of sleep related symptoms in four Latin American cities. J Clin Sleep Med 4, 579-585.

34. Jiménez-Genchi A \& Caraveo-Anduaga J (2017) Crude and adjusted prevalence of sleep complaints in Mexico City. Sleep Si 10, 113-121.

35. Moreno-Tamayo K, Manrique-Espinoza B, Ortiz-Barrios LB, et al. (2020) Insomnia, low sleep quality, and sleeping little are associated with frailty in Mexican women. Maturitas 136, 7-12.

36. Guerrero-Zúñiga S, Gaona-Pineda EB, Cuevas-Nasu L, et al. (2018) Prevalence of sleep symptoms and risk of obstructive sleep apnea in Mexico. Salud Publica Mex 60, 347-355.

37. Jansen EC, Baylin A, Cantoral A, et al. (2020) Dietary patterns in relation to prospective sleep duration and timing among Mexico City adolescents. Nutrients 12, 2305.

38. Jansen EC, Stern D, Monge A, et al. (2020) Healthier dietary patterns are associated with better sleep quality among midlife Mexican women. J Clin Sleep Med 16, 1321-1330.

39. Romero-Martínez M, Shamah-Levy T, Cuevas-Nasu L, et al. (2017) Diseño metodológico de la Encuesta Nacional de Salud y Nutrición de Medio Camino 2016. Salud Publica Mex 59, 299-305.

40. Watson NF, Badr MS, Belenky G, et al. (2015) Recommended amount of sleep for a healthy adult: a joint consensus statement of the American Academy of Sleep Medicine and Sleep Research Society. Sleep 38, 843-844.

41. American Academy of Sleep Medicine (2014) International Classification of Sleep Disorders, 3rd ed. Darien, IL: American Academy of Sleep Medicine.

42. Netzer NC, Stoohs RA, Netzer CM, et al. (1999) Using the Berlin questionnaire to identify patients at risk for the sleep apnea syndrome. Ann Intern Med 131, 485.

43. Institute of Medicine (2005) Dietary Reference Intakes for Energy, Carbobydrate, Fiber, Fat, Fatty Acids, Cholesterol, Protein, and Amino Acids. Washington, DC: National Academy Press.

44. Frankenfield D, Roth-Yousey L \& Compher C (2005) Comparison of predictive equations for resting metabolic rate in healthy nonobese and obese adults: a systematic review. J Am Diet Assoc 105, 775-789.

45. Thakkar MM, Sharma R \& Sahota P (2015) Alcohol disrupts sleep homeostasis. Alcohol 49, 299-310.

46. Weibel J, Lin Y-S, Landolt H-P, et al. (2020) Caffeine-dependent changes of sleep-wake regulation: evidence for adaptation after repeated intake. Prog Neuropsychopharmacol Biol Psychiatry 99, 109851.

47. Bailey RL, Gutschall MD, Mitchell DC, et al. (2006) Comparative strategies for using cluster analysis to assess dietary patterns. $J$ Am Diet Assoc 106, 1194-1200.

48. National Center for Health Statistics (2017) National Health Interview Survey - Adult Tobacco Use - Glossary. https://www.cdc.gov/nchs/ nhis/tobacco/tobacco_glossary.htm.

49. WHOEC on Physical Status (1993) Physical Status: The Use and Interpretation of Anthropometry, Report of a WHO Expert Committee. Geneva: World Health Organization.

50. Medina C, Barquera S \& Janssen I (2013) Validity and reliability of the international physical activity questionnaire among adults in Mexico. Rev Panam Salud Pública 34, 21-28.

51. Bland jM \& Altman DG (1995) Statistics notes: multiple significance tests: the Bonferroni method. Br Med J 310, 170-170.

52. Kim H \& Young T (2005) Subjective daytime sleepiness: dimensions and correlates in the general population. Sleep 28, 625-634.

53. Krishnan V \& Collop NA (2006) Gender differences in sleep disorders. Curr Opin Pulm Med 12, 383-389.

54. St-Onge M-P, Mikic A \& Pietrolungo CE (2016) Effects of diet on sleep quality. Adv Nutr 7, 938-949.

55. Lohsoonthorn V, Khidir H, Casillas G, et al. (2013) Sleep quality and sleep patterns in relation to consumption of energy drinks, 
caffeinated beverages, and other stimulants among Thai college students. Sleep Breath 17, 1017-1028.

56. Roehrs T \& Roth T (2001) Sleep, sleepiness, sleep disorders and alcohol use and abuse. Sleep Med Rev 5, 287-297.

57. Clark I \& Landolt HP (2017) Coffee, caffeine, and sleep: a systematic review of epidemiological studies and randomized controlled trials. Sleep Med Rev 31, 70-78.

58. Ehlers CL, Gilder DA, Criado JR, et al. (2010) Sleep quality and alcohol-use disorders in a select population of young-adult Mexican Americans. J Stud Alcohol Drugs 71, 879-884.

59. Vasquez MM (2008) Associations of dietary intake and physical activity with sleep disordered breathing in the Apnea Positive Pressure Long-Term Efficacy Study (APPLES). J Clin Sleep Med $4,8$.

60. Reid M, Maras JE, Shea S, et al. (2019) Association between diet quality and sleep apnea in the multi-ethnic study of atherosclerosis. Sleep 42, zsy194.

61. Barceló A, Barbé F, Llompart E, et al. (2005) Neuropeptide Y and leptin in patients with obstructive sleep apnea syndrome. Am Respir Crit Care Med 171, 183-187.
62. Shechter A, O'Keeffe M, Roberts AL, et al. (2012) Alterations in sleep architecture in response to experimental sleep curtailment are associated with signs of positive energy balance. Am J Physiol Integr Comp Physiol 303, R883-R889.

63. Frank S, Gonzalez K, Lee-Ang L, et al. (2017) Diet and sleep physiology: public health and clinical implications. Front Neurol 8, 393.

64. Araghi MH, Chen Y-F, Jagielski A, et al. (2013) Effectiveness of lifestyle interventions on obstructive sleep apnea (OSA): systematic review and meta-analysis. Sleep 36, 1553-1562.

65. Miller TM, Abdel-Maksoud MF, Crane LA, et al. (2008) Effects of social approval bias on self-reported fruit and vegetable consumption: a randomized controlled trial. Nutr J 7, 18.

66. Denova-Gutiérrez E, Ramírez-Silva I, Rodríguez-Ramírez S, et al. (2016) Validity of a food frequency questionnaire to assess food intake in Mexican adolescent and adult population. Salud Publica Mex 58, 617.

67. Cespedes EM \& Hu FB (2015) Dietary patterns: from nutritional epidemiologic analysis to national guidelines. Am J Clin Nutr 101, 899-900.

68. Hu F (2002) Dietary pattern analysis: a new direction in nutritional epidemiology. Curr Opin Lipidol 13, 3-9. 